\title{
A Cooperative and Competitive Workshop in Mechatronics Engineering
}

\author{
http://dx.doi.org/10.3991/ijep.v4i1.3068 \\ S. Krebs, S. Schmidt, S. Matthiesen and S. Hohmann \\ Karlsruhe Institute of Technology, Karlsruhe, Germany
}

\begin{abstract}
This paper presents a new mechatronics laboratory for students in the $5^{\text {th }}$ semester of the mechatronics degree course at the Karlsruhe Institute of Technology. It is the aim of this teaching event to sharpen the appreciation of synergy effects in the development of mechatronic systems among the students. Despite of the great freedom in the development process, a concept has been evolved, which causes low running costs due to the combination of a model kit with rapid prototyping methods. A first pilot study of the laboratory starting in the winter term 2014 has shown that the students approach the task despite of the high level of difficulty with fun and dedication, especially because of the wide solution space which was unknown for them from previous lectures.
\end{abstract}

Index Terms-Cooperation, mechatronics engineering, project-based learning, team decision making

\section{INTRODUCTION}

To meet the increasing integration of electronic and mechanical components and the resulting need for mechatronics engineers, a mechatronics degree course started at the Karlsruhe Institute of Technology in the winter term 2012. In the first four semesters, the students are taught in core competencies in the fields of electrical engineering, information technology and mechanical engineering, which are applied in the last semester before the thesis in a lecture with connected laboratory that is planned and carried out by the Department of Electrical Engineering and Information Technology as well as by the Department of Mechanical Engineering.

This new joint approach of two departments should convey the core idea of mechatronics, namely the use of synergy effects in the system development. As a part of this laboratory, the development process of a mechatronic system should be experienced under industrial conditions, which includes not only the use of standard development tools but also the distributed development with separate development teams.

In the following, the workshop developed for this purpose "Mechatronic systems and products" will be presented. After the already existing mechatronics workshops of other universities were introduced and judged briefly in chapter II the new idea of the presented workshop is described.

Since this workshop will take place in the winter term 2014 for the first time, a pilot study was conducted with students to provide an initial evaluation.

\section{EXISTING WORKSHOPS}

\section{A. Overview}

The usually conducted practical exercises in mechatronics courses can generally be divided into two groups. On the one hand, there are traditional laboratories in which a task has to be processed in a narrow solution framework. Under certain circumstances, even only one aspect of the mechatronics will be considered like the coding. In addition to this type of practical laboratories, there are also several workshops in which the participants receive a fixed task which can be solved with many open spaces. Instead of rigid experiment set-ups, these approaches use model kits and selfmade parts to not limit the creativity. In the following, some examples of both laboratories types will be introduced to be able to explain the novelty character of the workshop introduced here.

\section{B. Classical Workshops}

All the classical laboratories within the mechatronics have in common that a solid mechanical structure is present and the task only involves the programming of these systems.

This fact is owed of the history of these workshops: They are mostly descended from the Department of Mechanical Engineering of the university. It is presupposed that the mechanical engineering students already have sufficient mechanical knowledge and this must be enlarged merely with knowledge in the area of the electrical engineering and information technology. The main emphasis is on the control engineering and the programming mostly and the experience of synergy effects is ignored, on this occasion. An example for this is the mechatronics course at the Queen's University in Kingston [1]. In the laboratory organized by the Department of Mechanical Engineering, the students have to program a controller to hold a ping pong ball with the help of a fan at a certain level within a Plexiglas tube. As another practical example, a CIM cell is listed. However, this widespread example includes only the programming of a sequence and therefore does not serve to convey the core message of mechatronics, the synergy effect.

It behaves similarly at the program of the University of Minho [2]. Here, another classical application of the mechatronics is introduced, namely the robotics. Besides, there is another number of programming tasks, but due to the firmly predefined mechanics, these are similarly unsuitable.

An approach tending strongly in the direction of system modeling is introduced in [3]. Here, the predefined mechanical construction must be modeled in Matlab. After- 
wards, given controllers are used and the model is verified with measurements.

On the one hand, these three examples have in common that they are only organised by one department and, on the other hand, that the solution space for the students is very limited.

\section{Workshops with a wide solution framework}

The first publication which deals with practical exercises in mechatronics and lets freedom for the creativity simultaneously is [4]. The author argues explicitly herein for a task, which leaves enough free space in finding solutions, which have parts of software, electronics and mechanics (although the author teaches at the Department of Mechanical Engineering) and which shows a high level of difficulty to give the students a realistic impression of a development process.

In addition, other important information will be given, which are necessary for the development of the task. Particularly important, and to our knowledge not mentioned in another paper than the one given above, is the "coolness factor", because the enthusiasm for the subject is essential to find a solution for a difficult task. As an example, among others, an automatic golf putter is brought up.

The task is simply to transport five golf balls into a basket that is marked by a light. The solution space is completely open, which resulted besides others in autonomous vehicles.

Further tasks, that leave more freedom compared with the workshops presented in chapter II B and restrict the solutions, for example, only in the way that the solution has to be an autonomous vehicle, are [5], [6], [7] and [8].

\section{OBJECTIVES AND CONTENTS OF THE KIT'S WORKSHOP}

In contrast to the workshops presented in the previous chapters it is not our aim to educate engineers which can cover every area in a development process (construction, circuit design and programming) and are thus able to develop mechatronic products fully. These abilities have already been learned in the previous lectures and exercises.

The focus of this event is the targeted use of synergies in the development of new systems. The students should learn by an industry-oriented development process, that the link between the sub-areas of mechatronics produces better solutions than the optimal solution of one single sub-area. This procedure follows the interdisciplinary development teams in the industry.

\section{A. Statement of task}

In order to achieve the set objectives, a task with a wide solution space was chosen as it has been recommended in [4].

It is the aim of the development project to develop a complete system which gathers cubes and then builds these up to a tower in the field represented in figure 1. The system starts to this in the green area and builds up the tower in the red area. Besides the height of the tower, the time needed to built up the tower and to return to the green start area, counts for the point assignment. In addition to the white cubes, which are on the floor of the playing field, there is another green one on the passage for which there are bonus points and a red one with penalty points. Which one is on the right side and which one is on the left is unknown to force a color identification. The shape of the tower, as well as the strategy how it is built, is up to the team.

A first step in the direction of the industry near formulation is the division of the team which consists of ten students in two groups of five students each.

Each of these groups has to develop one independent subsystem. For the solution of the task, the cooperation of the two subsystems is then finally required.

Thus, the distributed development usual in the industry is trained.

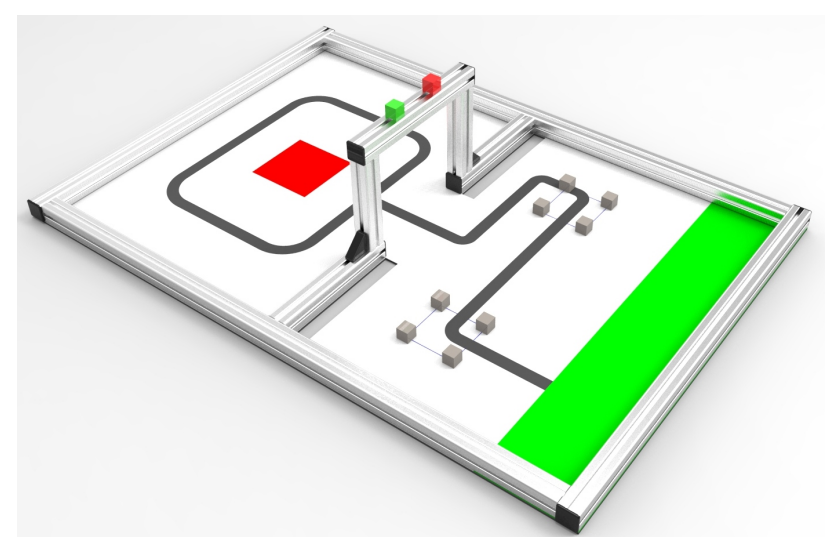

Figure 1. Playing field

\section{B. Milestone plan}

The milestone plan shown in figure 2 which contains the following points serves for the project planning and as a control possibility for the responsible persons of the workshop:

- $\quad$ Profile phase

○ Development of strategies for the fulfillment of the task

- Estimation of the maximum points available

- Identification of the critical points

- Idea phase

- Development of five solution ideas

- Determination of a preferred solution

- $\quad$ First milestone $\left(3^{\text {rd }}\right.$ week)

- Presentation of the developed strategy and of the five ideas

○ Reasoning the preferred solution

- Concept phase

- Estimation of the installation space

- Validation of critical points with experimental prototypes

- $\quad$ Second milestone $\left(6^{\text {th }}\right.$ week $)$

- Presentation of the results

- Detailing phase

- Detailing of the concepts

- Construction of the prototypes

- $\quad$ Third milestone $\left(10^{\text {th }}\right.$ week)

- Presentation of the prototypes

- Closing event (14 $4^{\text {th }}$ week)
- Group presentation
- Competition
- Award ceremony 

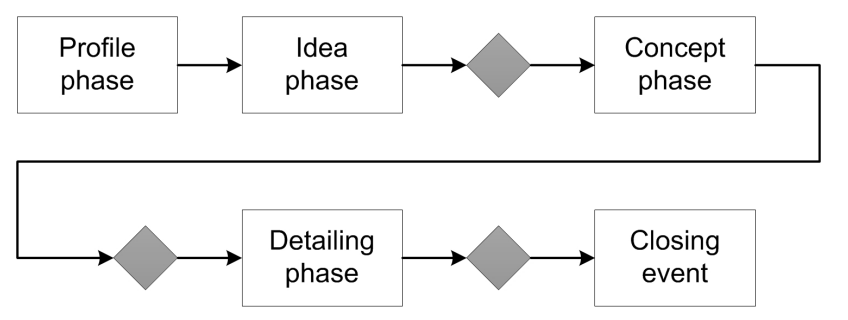

Figure 2. Milestone plan

In addition to the control and steering function of these milestones, they are also helpful for the students to focus their work on intermediate goals. So, one avoids that the teams work on the consideration for different concepts too long, for example and the time is therefore missing at the end to build up the prototypes. A very important point which already takes a large role at some planning ([4], [7]) is the closing event. The students present their results here in front of a large audience which is on the one hand an incentive to obtain a result as good as possible and on the other hand, it can be also interesting from the view of university marketing.

It is not a new idea to publish videos of such events on known platforms such as Youtube in order to attract potential students. The ETH Zurich pursues this approach for some time with the Innovation Project of the Autonomous Systems Lab.

\section{Resources}

At the planning of the resources for a workshop, on the one hand, the enabling of a wide space of solutions and on the other hand the economic feasibility which plays an important role in the university life contrast with themselves.

Firstly, the students should not be restricted by model kits, but simultaneously prototypes that consist of specially manufactured parts would be too expensive for the expected number of participants of more than 100 students every year.

How a compromise could be achieved between these two extremes taking into account a development close to the one in industry, should be shown in the following.

\section{Mechanical Resources}

Components of the company Fischertechnik, which could establish itself in the U.S. education sector (see [9]) provide the basic framework for the mechanical construction. Since there is a variety of different elements, it is possible to create a model kit with which the described task can be fulfilled without significant restrictions. Apart from the structural elements, the sensors and motors of Fischertechnik are also used.

However, such restrictions are also common in industrial development projects, since nearly everything is done here on already established standard components as the basis for new developments. For truly novel ideas, rapid prototyping methods are available in both, in industrial environments as well as in the presented workshop. In addition to a fused deposition modeling machine, students can use a 2D cutter to manufacture individual parts.

\section{E. Electronics/ Programming}

Minimal constraints on the development process should result also from the selected electronics and the program- ming environment and it should ensure an industry-related development. For this reason, the decision fell on an overperformant development board and a model-based software development. The development board is the TMS320F28335 Experimenter Kit of the company Texas Instruments (see figure 3). The used controlcard is equipped with a $150 \mathrm{MHz}$ floating point processor and is supported by the Embedded Coder per default in Matlab/ Simulink. This tool should be used for the code generation due to the awareness from previous lectures, and on the other hand, because it is a widely used tool for simulations in the industry.

After the program has been tested simulatively and by measurements with an oscilloscope, the controlcard is taken from the evaluationboard and inserted into a target hardware with motor drivers for $10 \mathrm{dc}$ motors and measuring bridges for the sensor evaluation.

This also overperformant target hardware is then integrated into the mechanical construction.

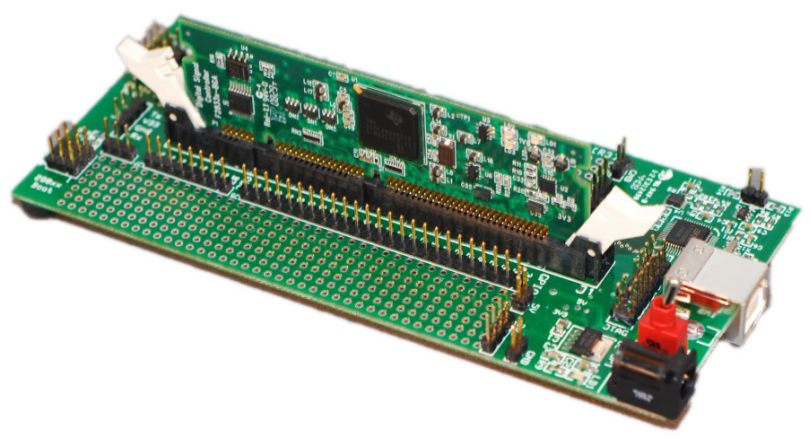

Figure 3. TMS320F28335 Experimenter Kit with controlcard [10]

\section{RESULTS OF THE FIRST TEST RUN}

Before the course was further developed, the concept has been evaluated in a pilot study with four students.

Two of them were from the Department of Mechanical Engineering and two from the Department of Electrical Engineering and Information Technology. These students were divided into two groups with one student of each subject area and should reach the first milestone within ten hours. Below, two of the resulting concepts are presented and subsequently the synergy effects on a specific problem are demonstrated.

\section{A. Concept 1: Slider-caterpillar with profile-vehicle}

The first concept involves the construction of two autonomous vehicles, each with different tasks.

To get the time bonus, the goal is to build a stable tower from $2 \times 4$ cubes and to fall below the required time. The cubes situated on the passage are ignored to this.

For this purpose, the first vehicle, shown in figure 4 on the right, pushes the cubes to a side band. Following, the complex vehicle shown on the left side of the figure takes over the building of the tower while the first vehicle already returns to the green zone. Afterwards, the second vehicle jams the cubes with the channel profile and drives to the red building area. Once there, it turns the profile by $90^{\circ}$, and opens it so that the tower is free. This vehicle then returns to the green area, too. 

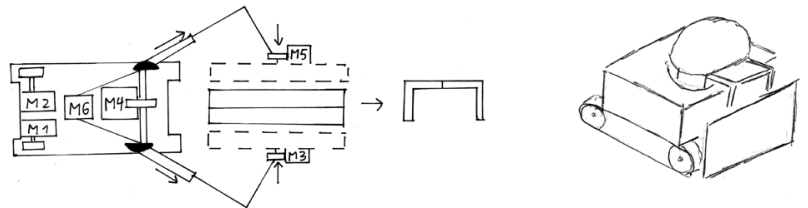

Figure 4. Profile concept

\section{B. Concept 2: Gantry crane with hopper vehicle}

The second concept aims to build a tower of maximum height $(1 \times 10)$ and to accept the time penalty. To achieve this goal, an autonomous vehicle which transports an openable hopper moves to the red construction area. Then, a gantry crane begins to pick up the cubes and to transport them into the hopper (see figure 5). Once all the cubes are in the hopper, this opens and the tower is set free. At the end, the gantry crane and the hopper car drive back into the green zone. To get in impression how such systems built with Fischertechnik can look like, the prototypes of the test run are illustrated in figure 6.
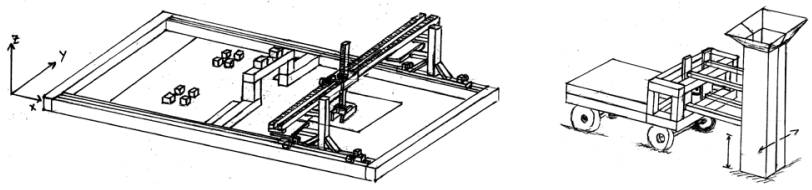

Figure 5. Crane Concept

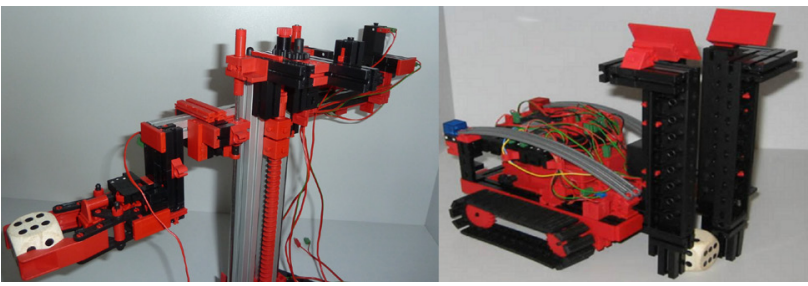

Figure 6. Prototypes of the crane concept

Just at the solution of this problem, it can be shown that there are for certain aspects strongly mechanically or strongly electrically (including sensors) distinct solutions.

An example of this is the orientation of the gantry crane at the hopper vehicle. The students have considered three concepts to this which are represented in the top view in figure 7. A sensorily very demanding solution which probably cannot be carried out with the sensors available is shown on the left. For the horizontal orientation, the color difference between the red hoppper opening and a laterally arranged plate having a green color gradient is used. The vertical orientation shall then be carried out with the help of the color gradient. However, in this solution not only a precise sensor is necessary, but also the mechanics must meet high standards in order to detect the gradient.

A sensorily less demanding solution is represented on the right edge of the figure. On the right, a bolt which is attached on a plate can be recognized in the top view. Furthermore, a socket which fits to the bolt has to be fixed to the crane. Once this connection has been detected, for example by a push button at the end of the socket, the orientation is complete. However, it can be difficult to accomplish this connection because the hopper's position is not exactly known. Therefore, a more complex socket, for example with a kind of a funnel, has to be constructed.
The third solution is shown in the middle. By means of a vertical and a horizontal bar code which is attached on a side plate and having the distance from the center of the bar code, the orientation of the hopper can be achieved with standard components such as the line sensor. This way, no higher request appears on the part of the mechanics than at the locomotion of the vehicles and the sensory task is also easier to solve than in the first concept.

However, it is important that the position resolution is selected through the available gears so that the barcode can be read.
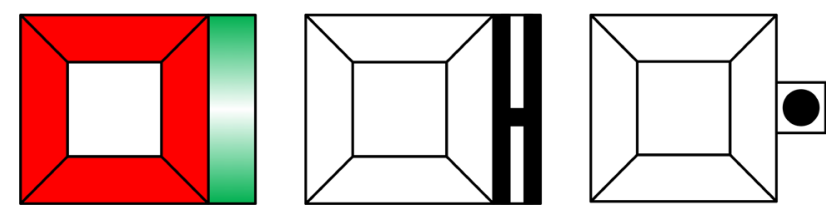

Figure 7. Concepts for the orientation of the gantry crane

\section{CONCLUSIONS AND FORECAST}

The proposed concept for a practical workshop at the end of a mechatronics degree program prepares students excellent for the development work in companies.

In addition to the understanding of synergy in the development of mechatronic systems, the usual distributed development will be trained by the collaboration of two teams.

Furthermore, it is made sure by reusable standard components combined with modern rapid prototype tools that the creativity of the students is not limited despite the minimal running costs.

At the further development of the teaching concept, we examine in the next step, whether a consideration of costs for the materials and the effort for the assembly proves to be sensible.

Moreover, considerations must be brought how the groups have to be split in order to achieve comparability of results.

\section{ACKNOWLEDGMENT}

We acknowledge support by Deutsche Forschungsgemeinschaft and Open Access Publishing Fund of Karlsruhe Institute of Technology.

Furthermore, we want to thank Fischertechnik for its support.

\section{REFERENCES}

[1] P. Wild, B. Surgenor, and G. Zak, "The Mechatronics laboratory experience", Mechatronics, vol. 12, pp. 207-215, 2002. http://dx.doi.org/10.1016/S0957-4158(01)00061-7

[2] M. Lima et al., "Mechatronics education at the University of Minho: a summary of the present; perspectives for the future", Mechatronics, vol. 12, pp. 295-302, 2002. http://dx.doi.org/10.1016/S0957-4158(01)00069-1

[3] X. E. Huang, "Extending Mechatronic Innovative and Practical Training Curriculum to Sophomore Unergraduates", Advanced Material Research, vol. 591-593, 2012.

[4] J. Carryer, "The Design Of Laboratory Experiments And Projects For Mechatronics Courses", Mechatronics, vol. 5, pp. 787-797, 1995. http://dx.doi.org/10.1016/0957-4158(95)00046-8

[5] S. G. Kibler et al., "IEEE Micromouse for Mechatronics Research and Education", Proceedings of the 2011 IEEE International Conference on Mechatronics, 2011. http://dx.doi.org/10.1109/ ICMECH.2011.5971240 
[6] T. Tokuyasu, "Installation of Mechatronics Education Using The MindStorms for Dept. of Mechanical Engineering O.N.C.T.", Proceedings of International Conference on Mechatronics, 2007

[7] W. Singhose et al., "Use of Design Competitions in Mechatronics Education", Proceedings of the 2009 IEEE International Conference on Mechatronics, 2009. http://dx.doi.org/10.1109/ ICMECH.2009.4957226

[8] S. E. Alpetkin, "Development of a Mechatronics Design Studio", Industrial and Manufactoring Engineering Department, Cal Poly, San Luis Obispo, 1997

[9] Fischertechnik GmbH, URL: http://fischertechnikstemlab.org/

[10] Texas Instruments Incorporated, URL: http://www.ti.com/ graphics/tool/f28335 usb dock.jpg

\section{AUTHORS}

S. Krebs is with the Department of Electrical Engineering and Information Technology, Karlsruhe Institute of Technology, Karlsruhe, Germany.

S. Schmidt is with the Department of Mechanical Engineering, Karlsruhe Institute of Technology, Karlsruhe, Germany.

S. Matthiesen is with the Department of Mechanical Engineering, Karlsruhe Institute of Technology, Karlsruhe, Germany.

S. Hohmann is with the Department of Electrical Engineering and Information Technology, Karlsruhe Institute of Technology, Karlsruhe, Germany.

Submitted 29 July 2013. Published as re-submitted by the authors 23 January 2014. 